\title{
A Microring Temperature Sensor Based on the Surface Plasmon Wave
}

\author{
Wenchao Li, ${ }^{1}$ Xiaopeng Sha, ${ }^{1}$ Dongyang An, ${ }^{2}$ and Zhiquan $\mathrm{Li}^{2}$ \\ ${ }^{1}$ School of Control Engineering, Northeastern University at Qinhuangdao, Qinhuangdao 066004, China \\ ${ }^{2}$ Institute of Electrical Engineering, Yanshan University, Qinhuangdao 066004, China
}

Correspondence should be addressed to Xiaopeng Sha; lhh120116@163.com

Received 5 November 2014; Revised 19 January 2015; Accepted 22 January 2015

Academic Editor: Jayanta K. Sahu

Copyright (C) 2015 Wenchao Li et al. This is an open access article distributed under the Creative Commons Attribution License, which permits unrestricted use, distribution, and reproduction in any medium, provided the original work is properly cited.

A structure of microring sensor suitable for temperature measurement based on the surface plasmon wave is put forward in this paper. The sensor uses surface plasmon multilayer waveguiding structure in the vertical direction and U-shaped microring structure in the horizontal direction and utilizes SOI as the thermal material. The transfer function derivation of the structure of surface plasmon microring sensor is according to the transfer matrix method. While the change of refractive index of Si is caused by the change of ambient temperature, the effective refractive index of the multilayer waveguiding structure is changed, resulting in the drifting of the sensor output spectrum. This paper focuses on the transmission characteristics of multilayer waveguide structure and the impact on the output spectrum caused by refractive index changes in temperature parts. According to the calculation and simulation, the transmission performance of the structure is stable and the sensitivity is good. The resonance wavelength shift can reach $0.007 \mu \mathrm{m}$ when the temperature is increased by $100 \mathrm{k}$ and FSR can reach about $60 \mathrm{~nm}$. This structure achieves a high sensitivity in the temperature sense taking into account a wide range of filter frequency selections, providing a theoretical basis for the preparation of microoptics.

\section{Introduction}

Conventional sensors are huge, even with mechanical components. Miniature temperature sensors have breakthroughs in terms of miniaturization and intelligence. Compared with the biosensing [1], piezoelectric sensing [2], optical sensing [3], and other sensing technologies, the surface plasmon (surface plasmon, SP) sensor has the advantage of small size, simple operation, high sensitivity, label-free, nondestructive, online real-time remote monitoring, and so forth. But there are few reports on temperature sensor which combines surface plasmon wave with microring structure.

The idea that microring resonator can be used in sensor was proposed by Marcatili in 1969 [4]. It is applied in a wide range of areas such as delay [5], filtering [6], lasers [7], and optical switching [8]. In recent years, the sensor with microring has excellent performance in the field of measuring pressure [9], acceleration [10], temperature [11], and gas density [12] and is especially widely used in microsize temperature sensor as the waveguide sensitive material [13].
SOI material, "silicon-on-insulator" for short, which has the advantage of low power, high speed, high integration, high temperature resistance, and antiradiation, making itself an extremely wide range of applications, is internationally recognized as the next generation of silicon-based integrated materials [14].

Zhang et al. proposed a plasma structure having five layers of $A B C B A$ in 2010, providing the theoretical preparation for the applications of multisurface plasma structure in the aspect of surface acoustic wave sensor [15]. Kong et al. proposed a plasma structure having five layers of MLHLM in 2012. It has better local capacity compared with the MIM (metal-insulator-metal) having the same thickness of insulation and can support the mode whose propagation length is up to $10^{3} \mu \mathrm{m}$ and quality factor is up to $10^{4}[16]$. This paper presents a novel microring temperature sensor based on surface plasma. This sensor has good sensitivity and the resonance wavelength shift can reach $0.007 \mu \mathrm{m}$ when temperature is increased by $100 \mathrm{k}$ and FSR can reach about $60 \mathrm{~nm}$. This paper discusses the influence of temperature 


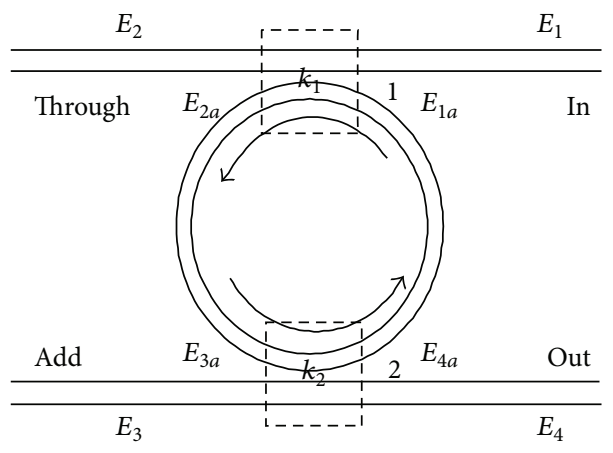

(a)

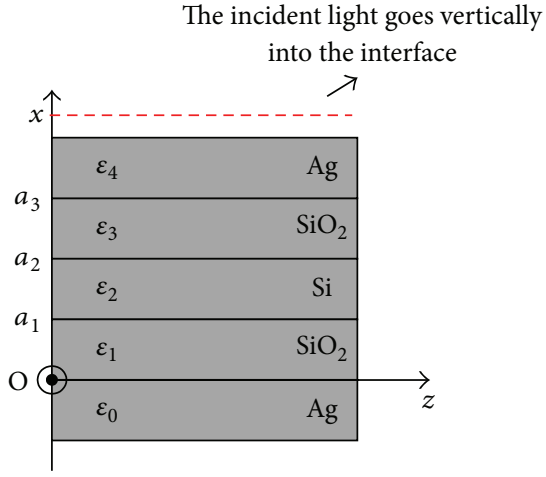

(b)

FIGURE 1: (a) Cross sectional structure chart of the microring temperature sensor based on surface plasmon; (b) vertical sectional structure of the microring temperature sensor based on surface plasmon.

changes in the external environment on the internal system parameters and analyzes the stability of transmission system so as to provide a theoretical basis for the preparation of temperature sensor and microoptics device.

\section{Model Building and Theoretical Analysis}

2.1. The Effective Refractive Index of the Surface Plasmon Structure. The establishment of a Cartesian coordinate system is shown in Figure 1(b). The propagation is assumed along the direction of $z \cdot x=0, a_{1}, a_{2}, a_{3}$ are the boundaries. The electromagnetic field may be represented as $E=\left(E_{x}, 0, E_{z}\right) e^{i(\beta z-\omega t)}$, $H=\left(1 / \mu_{0} c\right)\left(0, H_{y}, 0\right) e^{i(\beta z-\omega t)} \cdot \beta, \omega$, and $c$, respectively, are the propagation constant, the angular frequency, magnetic permeability of vacuum, and the velocity of light in vacuum.

The expression of $E$ and $H$ can be obtained by solving the vector wave equation in the boundary conditions of the tang component of the electromagnetic field being continuous. The unique solution of wave equations can be guaranteed by the Helmholtz and $n_{\text {eff }}$ theory, and it can be obtained by solving the following equation [16]:

$$
\begin{aligned}
0= & \left(N_{01} N_{23}+N_{12} N_{34}\right) T_{1} T_{2} T_{3}+\left(N_{12}+N_{01} N_{34}\right) T_{1} T_{2} \\
& +\left(N_{13}+N_{01} N_{34}\right) T_{1} T_{3}+\left(N_{23}+N_{02} N_{34}\right) T_{2} T_{3} \\
& +\left(N_{01}+N_{14}\right) T_{1}+\left(N_{02} N_{24}\right) T_{2}+\left(N_{03}+N_{34}\right) T_{3} \\
& +\left(1+N_{04}\right),
\end{aligned}
$$

where

$$
E_{x}= \begin{cases}-i A \frac{n_{\mathrm{eff}} k_{0}}{U_{0}} e^{x u_{0}}, & x \leq 0, \\ -i \frac{n_{\mathrm{eff}} k_{0}}{U_{p}}\left[A_{p 1} \cosh \left(U_{p} x\right)+A_{p 2} \sinh \left(U_{p} x\right)\right], & a_{p-1} \leq x \leq a_{p},(p=1,2,3), \\ i A_{4} \frac{n_{\mathrm{eff}} k_{0}}{U_{4}} e^{-U_{4}\left(x-a_{3}\right)}, & x \geq a,\end{cases}
$$

$$
\begin{aligned}
& E_{z}= \begin{cases}A e^{u_{0} x}, & x \leq 0, \\
A_{p 1} \sinh \left(u_{p} x\right)+A_{p 2} \cosh \left(u_{p} x\right), & a_{p-1} \leq x \leq a_{p}, \\
& (p=1,2,3), \\
A_{4} e^{-u_{4}\left(x-a_{3}\right)}, & x \geq a,\end{cases} \\
& H_{y}= \begin{cases}-i A \frac{\varepsilon_{0} k_{0}}{u_{0}} e^{u_{0} x}, & x \leq 0, \\
-i \frac{\varepsilon_{0} k_{0}}{u_{p}}\left[A_{p 1} \cosh \left(u_{p} x\right)+A_{p 2} \sinh \left(u_{p} x\right)\right], & a_{p-1} \leq x \leq a_{p},(p=1,2,3) \\
i A_{4} \frac{\varepsilon_{4} k_{0}}{u_{4}} e^{-u_{4}\left(x-a_{3}\right)}, & x \geq a,\end{cases} \\
& {\left[\begin{array}{l}
A_{11} \\
A_{12}
\end{array}\right]=\left[\begin{array}{c}
N_{01} \\
1
\end{array}\right] A,} \\
& {\left[\begin{array}{l}
A_{31} \\
A_{32}
\end{array}\right]=\left[\begin{array}{cc}
-S_{23} & C_{32} \\
C_{23} & -S_{23}
\end{array}\right]\left[\begin{array}{cc}
S_{22} & C_{22} \\
N_{23} C_{22} & N_{23} S_{22}
\end{array}\right]\left[\begin{array}{l}
A_{21} \\
A_{22}
\end{array}\right] \text {, }} \\
& {\left[\begin{array}{l}
A_{21} \\
A_{22}
\end{array}\right]=\left[\begin{array}{cc}
-S_{12} & C_{12} \\
C_{12} & -S_{12}
\end{array}\right]\left[\begin{array}{cc}
S_{11} & C_{11} \\
N_{12} C_{11} & N_{12} S_{11}
\end{array}\right]\left[\begin{array}{l}
A_{11} \\
A_{12}
\end{array}\right] \text {, }} \\
& {\left[\begin{array}{l}
A_{4} \\
A_{4}
\end{array}\right]=\left[\begin{array}{cc}
S_{33} & C_{33} \\
-N_{34} C_{33} & -N_{34} S_{33}
\end{array}\right]\left[\begin{array}{l}
A_{31} \\
A_{32}
\end{array}\right] \text {. }}
\end{aligned}
$$

In the equation, $S_{p q}=\sinh \left(a_{p}, u_{p}\right), C_{p q}=\cosh \left(a_{p} u_{p}\right)$, $N_{p q}=\varepsilon_{p} u_{q} / \varepsilon_{q} u_{p}, T_{p}=\tanh \left(d_{p} u_{p}\right), u_{p}=k_{0}\left(n_{\text {eff }}^{2}-\varepsilon_{p}\right)^{1 / 2}, k_{0}$ is the vacuum wave vector, $n_{\text {eff }}=\beta / k_{0}$ is the effective refractive index, and $p, q=0,1,2,3,4$.

\subsection{The Transfer Function of Microring Surface Plasmon} Sensor. This paper presents a microring temperature sensor based on surface plasmon, shown in Figure 1(a). $E_{i}$ is the complex amplitude of light field of each port $\left(E_{3}=0\right) . I_{i}$ is light field intensity. $I_{i}=\left|E_{i}\right|^{2}$ ( $i$ represents $1,2,3$, and 4$) . n_{\text {eff }}$ 
is the effective refractive index of the medium. The complex amplitudes of light field at the first coupling region and the second coupling region, respectively, are $E_{1 a}, E_{2 a}, E_{3 a}, E_{4 a}$. $k_{1}$ is the coupling coefficient between the microring and waveguide and $t_{1}$ is the transmission coefficient in the first coupling region. $k_{2}$ is the coupling coefficient between the microring and waveguide and $t_{2}$ is the transmission coefficient in the second coupling region. Coupling coefficients and transmission coefficients both meet the conditions of $k^{2}+t^{2}=$ 1. Loss factor of light transmission for a cycle in the microring is $\tau=\exp (-\alpha L) . \alpha$ is the loss coefficient of the microring. $L=2 \pi R$ is the circumference of the microring. ( $R$ is the radius of the microring.) $\tau=\exp (-a L)$ is the phase factor of light transmission in the microring a half week. The phase change of light transmission for semicycle is $\phi=\pi L n_{\text {eff }} / \lambda_{m}$ ( $\lambda_{m}$ is the resonant wavelength). According to the transfer matrix method [17], it can be drawn as follows:

$$
\begin{aligned}
& {\left[\begin{array}{c}
E_{1 a} \\
E_{2 a}
\end{array}\right]=\left[\begin{array}{cc}
\frac{-t_{1}}{i k_{1}} & \frac{1}{i k_{1}} \\
\frac{-1}{i k_{1}} & \frac{t_{1}}{i k_{1}}
\end{array}\right]\left[\begin{array}{l}
E_{1} \\
E_{2}
\end{array}\right]=M_{1}\left[\begin{array}{l}
E_{1} \\
E_{2}
\end{array}\right],} \\
& {\left[\begin{array}{c}
E_{3 a} \\
E_{4 a}
\end{array}\right]=\left[\begin{array}{cc}
0 & \tau^{1 / 2} p \\
\left(\tau^{1 / 2} p\right)^{-1} & 0
\end{array}\right]\left[\begin{array}{l}
E_{1 a} \\
E_{2 a}
\end{array}\right]=M_{t}\left[\begin{array}{l}
E_{1 a} \\
E_{2 a}
\end{array}\right],} \\
& {\left[\begin{array}{l}
E_{3} \\
E_{4}
\end{array}\right]=\left[\begin{array}{cc}
\frac{-t_{2}}{i k_{2}} & \frac{1}{i k_{2}} \\
\frac{-1}{i k_{2}} & \frac{t_{1}}{i k_{2}}
\end{array}\right]\left[\begin{array}{c}
E_{3 a} \\
E_{4 a}
\end{array}\right]=M_{2}\left[\begin{array}{c}
E_{3 a} \\
E_{4 a}
\end{array}\right] \text {, }} \\
& {\left[\begin{array}{l}
E_{3} \\
E_{4}
\end{array}\right]=M_{2} M_{t 1} M_{1}\left[\begin{array}{l}
E_{1} \\
E_{2}
\end{array}\right]} \\
& =\left[\begin{array}{ll}
\frac{t_{1}-t_{2} \tau p^{2}}{k_{1} k_{2} p \tau^{1 / 2}} & \frac{-1+t_{1} t_{2} \tau p^{2}}{k_{1} k_{2} p \tau^{1 / 2}} \\
\frac{t_{1} t_{2}-\tau p^{2}}{k_{1} k_{2} p \tau^{1 / 2}} & \frac{-t_{2}+t_{1} \tau p^{2}}{k_{1} k_{2} p \tau^{1 / 2}}
\end{array}\right]\left[\begin{array}{l}
E_{1} \\
E_{2}
\end{array}\right] .
\end{aligned}
$$

Since $E_{3}=0$, the amplitude transfer function $T$ and spectral intensity transfer function $I_{t}$ of the Out port are, respectively,

$$
\begin{gathered}
T=\frac{E_{4}}{E_{1}}=\frac{-k_{1} k_{2} \tau^{1 / 2} p}{1-t_{1} t_{2} \tau p^{2}}, \\
I_{t}=\left(\frac{E_{4}}{E_{1}}\right)^{2}=\frac{k_{1}^{2} k_{2}^{2} \tau^{2} p^{2}}{\left(1-t_{1} t_{2} \tau p^{2}\right)^{2}} .
\end{gathered}
$$

TABLE 1: The dielectric constants of different material.

\begin{tabular}{lcc}
\hline Material & Dielectric constant $\varepsilon$ & Thickness $(\mathrm{nm})$ \\
\hline $\mathrm{Ag}$ & $-126+2.9 i$ & 200 \\
\hline $\mathrm{SiO}_{2}$ & 1.44 & 180 \\
\hline & $3.4(T=298 \mathrm{k})$ & \\
$\mathrm{Si}$ & $3.363(T=498 \mathrm{k})$ & 180 \\
& $3.326(T=698 \mathrm{k})$ & \\
& $3.288(T=898 \mathrm{k})$ & \\
\hline
\end{tabular}

The amplitude transfer function $B$ and spectral intensity transfer function $I_{b}$ of the Through port are, respectively,

$$
\begin{gathered}
B=\frac{E_{2}}{E_{1}}=\frac{t_{1}-t_{2} \tau p^{2}}{1-t_{1} t_{2} \tau p^{2}}, \\
I_{b}=\left(\frac{E_{2}}{E_{1}}\right)=\left[\frac{\left(t_{1}-t_{2} \tau p^{2}\right)}{1-t_{1} t_{2} \tau p^{2}}\right]^{2} .
\end{gathered}
$$

\section{Simulation Analysis and Discussion}

3.1. Electric Field Analysis. To analyze the electric field distribution of the structure of the surface plasmon sensor, this paper uses the finite element method in Figure 1(a). The electric field distributions are shown in Figures 2(a), 2(b), 2(c), and 2(d) when temperatures are, respectively, $T=298 \mathrm{k}$, $T=498 \mathrm{k}, T=698 \mathrm{k}$, and $T=898 \mathrm{k}$. The incident light $(\lambda=1550 \mathrm{~nm}$ nonresonant wavelength) passes the layer silver Ag, silica dioxide $\mathrm{SiO}_{2}$, silicon $\mathrm{Si}$, silicon dioxide $\mathrm{SiO}_{2}$, and silver Ag successively from left to right. The different material properties of each layer are shown in Table 1.

The distance when the electromagnetic energy is attenuated to the initial value's $1 / e$ is defined as transmission length $L_{p} . L_{p}=1 / 2 I_{m}(\beta)$. The maximum transmission distance $L_{p}$ can reach $4719 \mu \mathrm{m}$ in the metal-low refractive index, high refractive index, low refractive index, and metal structure [17]. It meets the transfer requirements of new microring surface plasma temperature sensor designed in this paper in Figure 1(a).

$L_{p}$ is deeply influenced by the core. Replacing the inner core material, changing the refractive index in the core material, and increasing or decreasing the thickness of the inner core material can lead to corresponding change of $L_{p}$. $L_{\mathrm{Si}}$ can be increased by adjusting the thickness of the silica layer and Si layer in the structure designed in this paper.

It can be drawn from four temperature field distribution diagrams in Figure 2. When the nonresonant wavelength of the incident light wave $\lambda=1550 \mathrm{~nm}$, the electric field distribution in different temperature is a significant local result. The high energy region of the electric field is basically distributed in the sensing layer, Si layer, of the surface plasmon sensor, appearing to be a significant localized phenomenon.

Figure 3 reflects relationship between the temperature and the peak of electric field strength in Si layer. When the ambient temperature varies in the range of $T=298-1098 \mathrm{k}$, 

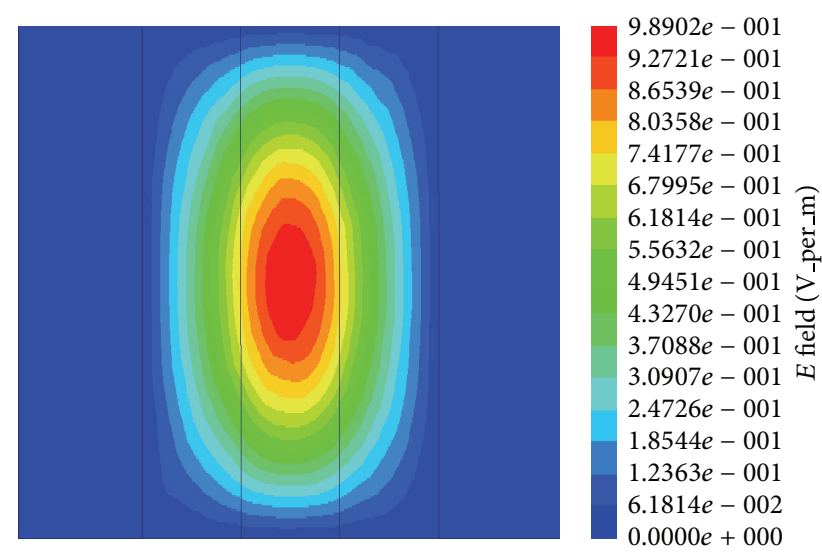

(a)
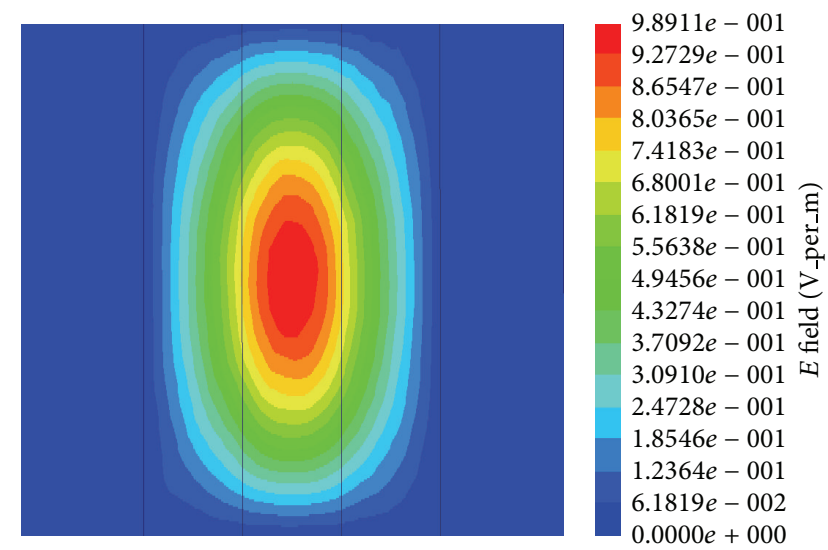

(c)

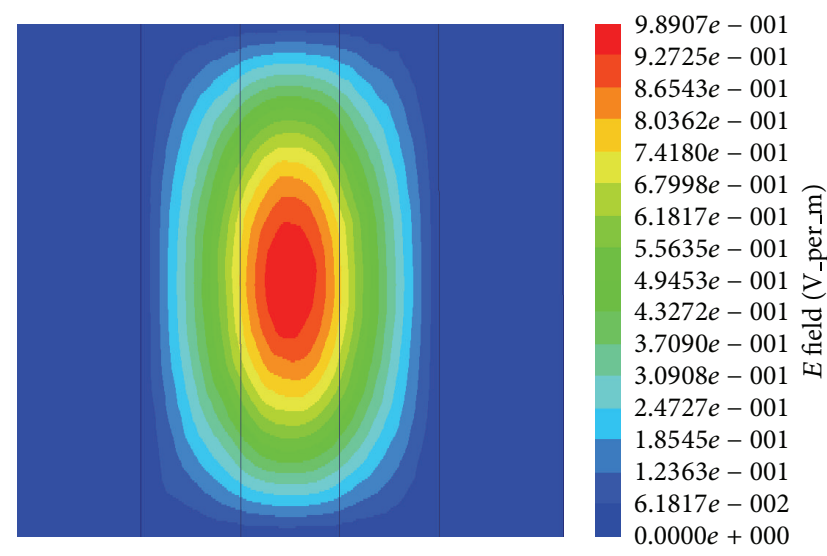

(b)

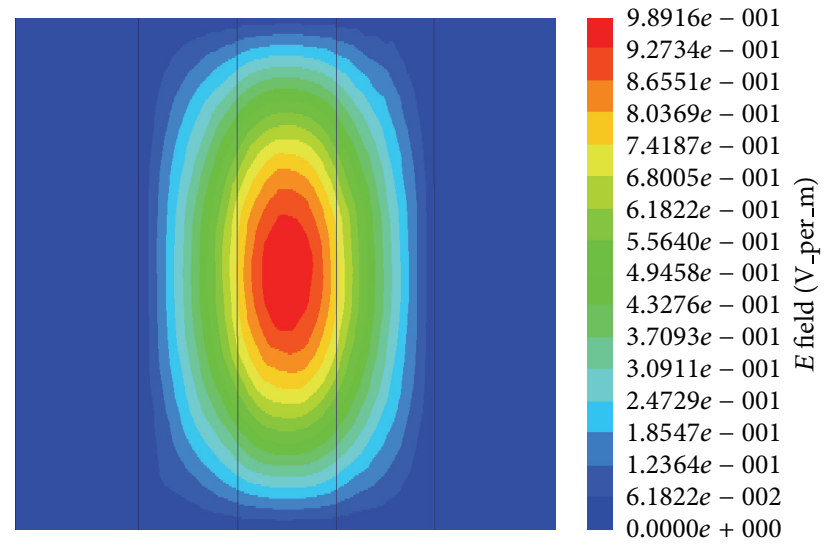

(d)

FIgURE 2: The electric field distribution. (a) $T=298 \mathrm{k}$; (b) $T=498 \mathrm{k}$; (c) $T=698 \mathrm{k}$; (d) $T=898 \mathrm{k}$.

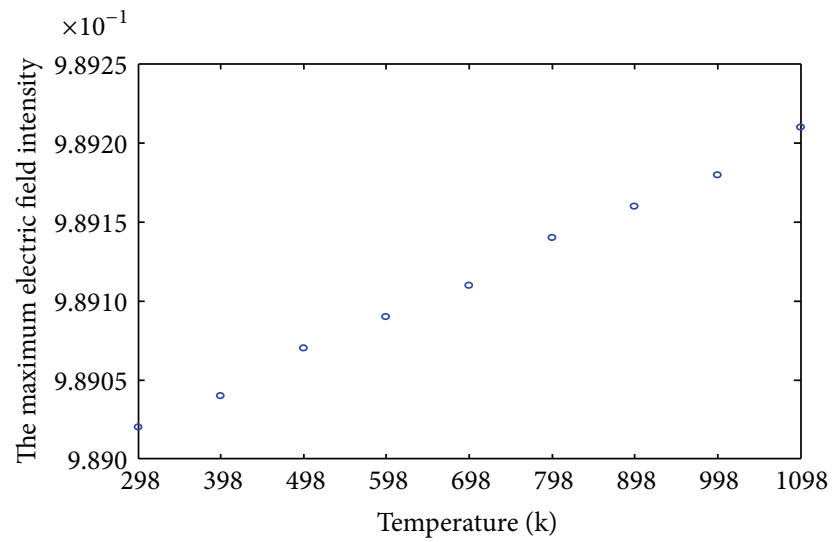

FIgURE 3: The relationship between the temperature and the peak of electric field strength of the Si layer.

the temperature and the maximum field strength of Si layer is substantially linear; when the temperature $T$ equals $298 \mathrm{k}$ $(T=298 \mathrm{k})$, the electric field strength reaches the minimum value $9.8902 e-001(e=9.8902 e-001)$; when the temperature $T$ equals $898 \mathrm{k}(T=898 \mathrm{k})$, the electric field strength reaches the maximum value 9.8921e-001 $(e=9.8921 e-001)$. It can guarantee the stability of the new microring surface plasmon sensor's transmission performance so that the sensor can be well applied in the field of sensors.

3.2. Relationship between the Effective Refractive Index and the Ambient Temperature. In optical waveguide, the temperature 


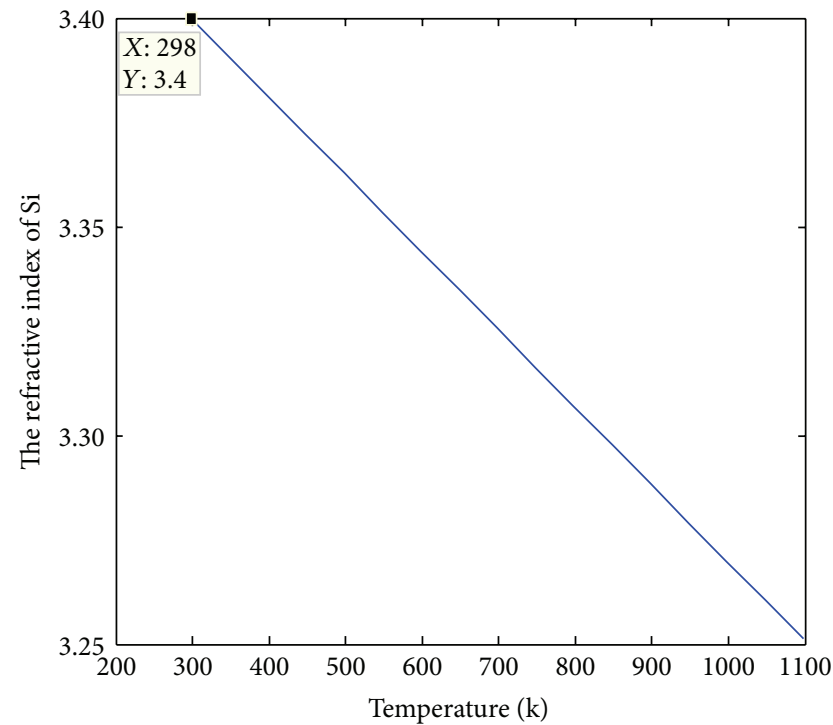

(a)

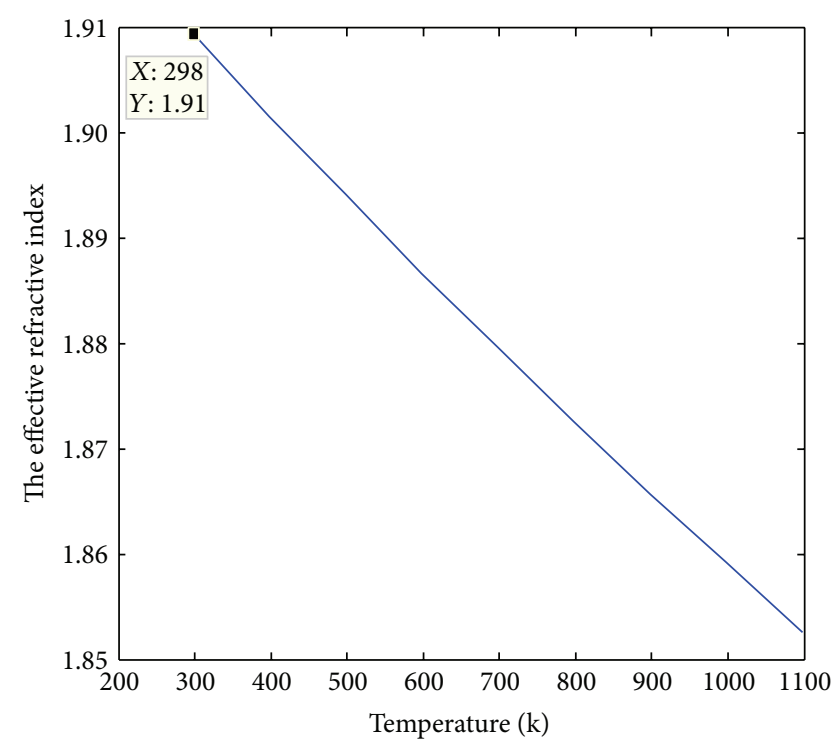

(b)

Figure 4: (a) The relationship between temperature and $n_{\mathrm{Si}}$. (b) The relationship between temperature and $n_{\text {eff }}$.

rise may cause thermal expansion of the material of $\mathrm{Si}$, thereby causing a structural change. The structure designed in this paper can ignore expansion caused by temperature rise and merely consider the influence on the refractive index $\left(n_{\mathrm{Si}}\right)$ of Si material and the effective refractive index of surface plasmon microring temperature sensor because of temperature rise since the metal is less affected by temperature and SPP wave produces at the contact area between $\mathrm{Ag}$ and $\mathrm{SiO}_{2}$. $n_{\mathrm{Si}}=3.4$ is Sis refractive index at room temperature $T=$ $298 \mathrm{k}$. Si has a large negative thermal coefficient of about $-1.86 \times 10^{-4} / \mathrm{k}$, so the refractive index of silicon $\mathrm{Si}$ and the relationship between structure of surface plasmon microring temperature sensor and temperature can be derived by (1). We get that the refractive index of silicon $\mathrm{Si}$ and the effective refractive index of surface plasmon microring temperature sensor and temperature $(T)$ appear to have an approximately linear relationship as shown in Figure 4. In Figure 4(b), the horizontal axis represents the temperature of Si layer, and the three curves show the effective refractive index of the whole waveguide. The curves show that the refractive index barely changed with the thermal expansion. Thus the influence from thermal expansion is not remarkable, which could be ignored.

3.3. Sensing Characteristic Analysis. The coupling coefficients of the surface plasmon microring temperature sensor designed in this paper meet the conditions of $k=0.018, L_{1}=$ $2 L$, and $R=4 \mu \mathrm{m}$. The outputs spectrum of Out port and Through port under $T=298 \mathrm{k}, 498 \mathrm{k}, 698 \mathrm{k}$, and $898 \mathrm{k}$ cases are shown in Figure 3. The Out port is the primary sensor output port. The Through port is the auxiliary sensor output port and is able to demonstrate the results of the outputs spectrum of Out port. Red curve (1) represents $I_{t}$. Black curve (2) represents $I_{b}$.
As shown in Figure 5, the output spectrum has good light extinction effect and has obvious spectral shift at four cases of $T=298 \mathrm{k}, 498 \mathrm{k}, 698 \mathrm{k}, 898 \mathrm{k}$. Temperature measurement can be achieved; then the FSR reaches $60 \mathrm{~nm}$.

Relationship between the temperature and the amount of shift of the resonance wavelength is shown in Figure 6. We can achieve the temperature sensor by detecting the resonance wavelength shifting. The displacement of the resonant peak is $0.007 \mu \mathrm{m}$ when temperature is increased by $100 \mathrm{k}$. The relationship between the resonance wavelength shift and the amount of temperature change is significantly linear. The range is larger. It suits temperature sensing.

\section{Conclusion}

The sensor put forward in this paper uses surface plasmon multilayer waveguide structure in the vertical direction and U-shaped microring structure in the horizontal direction and utilizes SOI as the thermal material. Combining with surface plasmon transmission technology and through the theoretical analysis of output spectrum at different temperatures and analysis of the intensity distribution of the structure, this paper comes up with a surface plasmon microring temperature sensor with stable transmission and high sensitivity. The resonance wavelength shift can reach $0.007 \mu \mathrm{m}$ when temperature is increased by $100 \mathrm{k}$ and FSR can reach about $60 \mathrm{~nm}$, achieving a high sensitivity in the temperature sense taking into account a wide range of filter frequency selections. The transmission performance of the structure is stable. This paper presents and analyzes a new type of surface plasmon sensor, providing theoretical innovations for the production of high-sensitivity temperature sensor and broadening the theoretical basis of surface plasmon. 


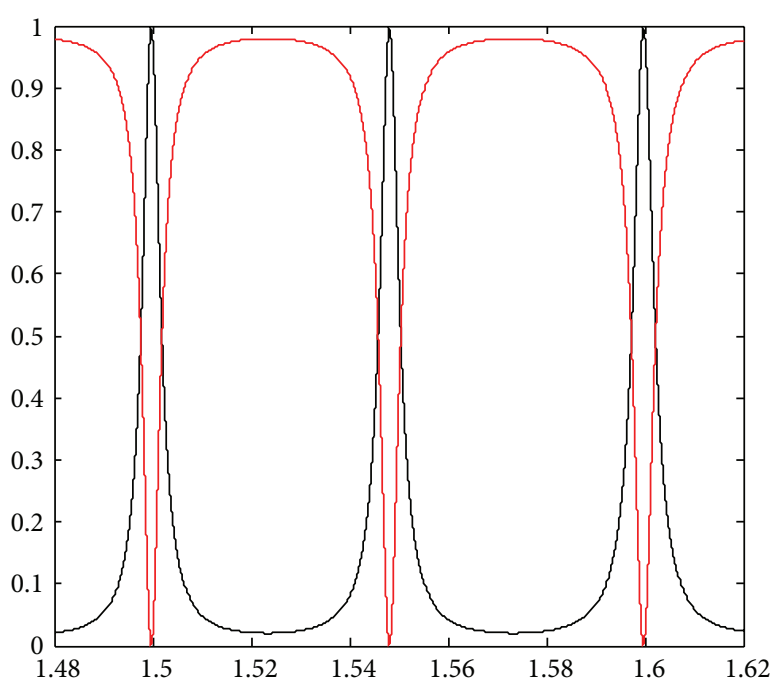

(a)

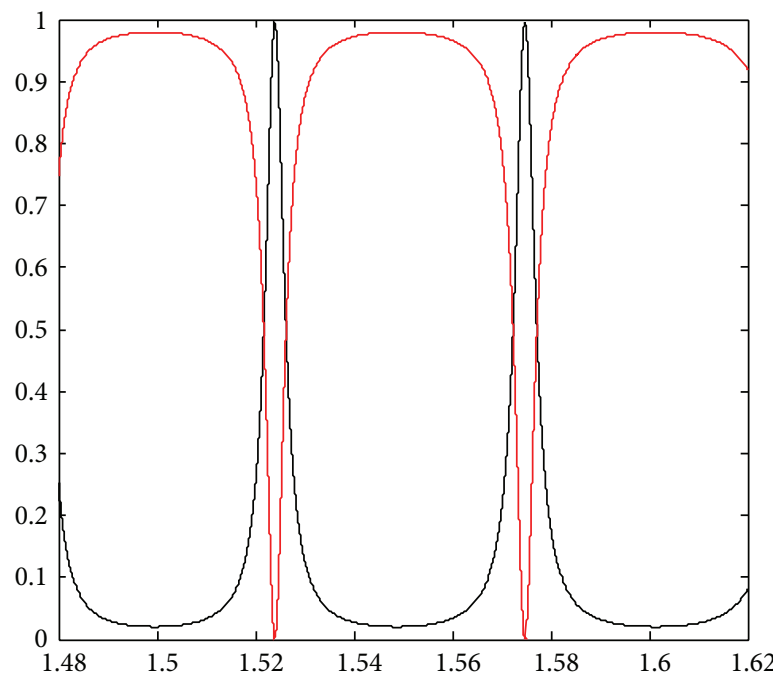

(c)

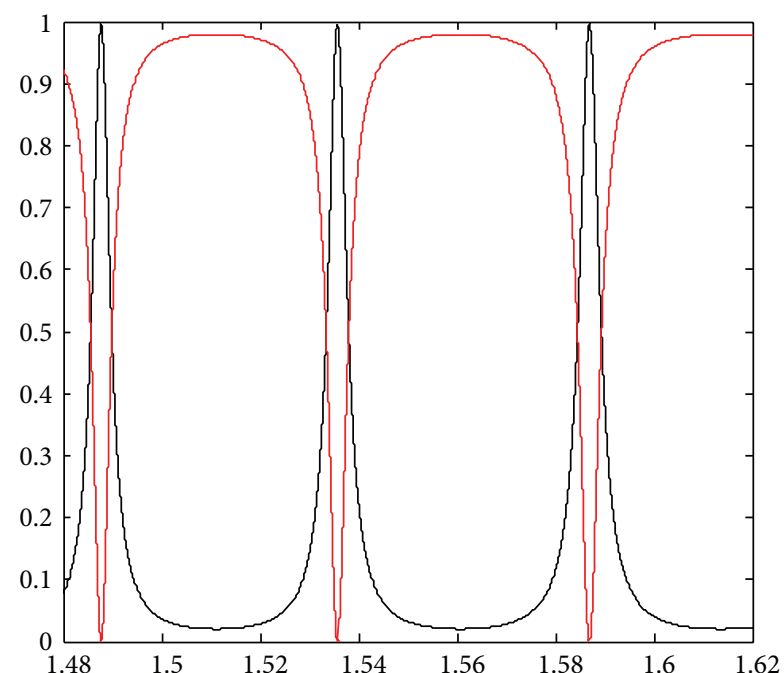

(b)

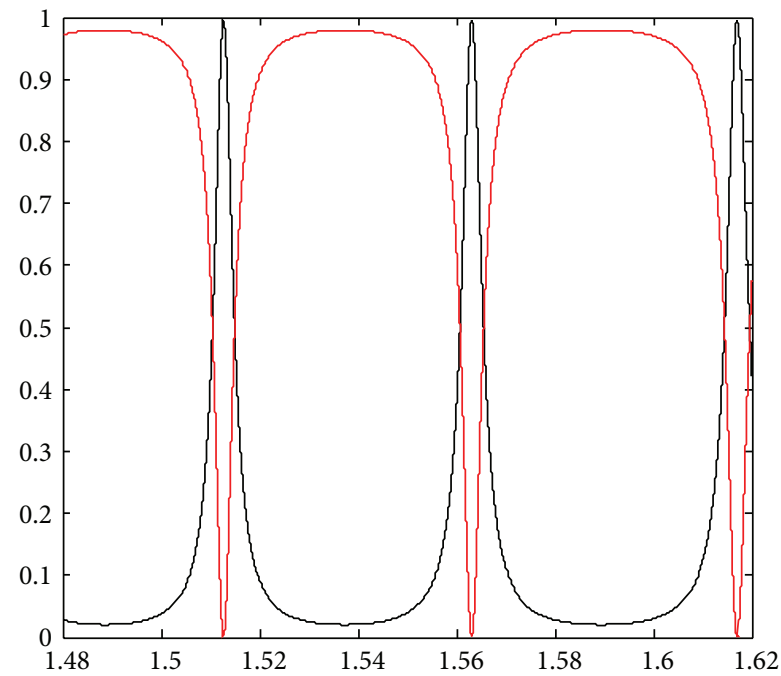

(d)

Figure 5: Output spectrum. (a) $T=298 \mathrm{k}$; (b) $T=498 \mathrm{k}$; (c) $T=698 \mathrm{k}$; (d) $T=898 \mathrm{k}$.

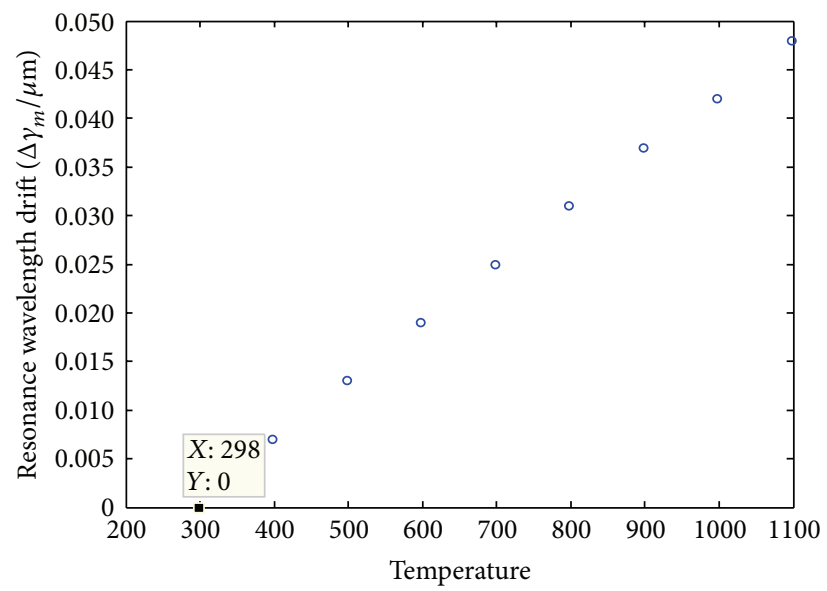

Figure 6: Relations between resonance wavelength shift and temperature. 


\section{Conflict of Interests}

The authors declare that there is no conflict of interests regarding the publication of this paper.

\section{Acknowledgments}

This work was supported by the Natural Science Foundation of Hebei Province (Grant no. F2014501150) and the NEUQ supporting-technology program under Grant no. XNK201405.

\section{References}

[1] J. J. Cheng, J. D. Li, B. Miao et al., "Ultrasensitive detection of $\mathrm{Hg}^{2+}$ using oligonucleotide-functionalized AlGaN/GaN high electron mobility transistor," Applied Physics Letters, vol. 105, no. 8, Article ID 083121, 4 pages, 2014.

[2] X. Y. Ma, K. A. Chen, S. H. Ding, and B. R. Zhang, "Optimization of piezoelectric sensor arrays in error sensing of active triple sound insulation structure," Acta Physica Sinica, vol. 62, no. 12, Article ID 124301, 2013.

[3] M. H. Tyboroski, N. R. Anderson, and R. E. Camley, "An effective medium study of surface plasmon polaritons in nanostructured gratings using attenuated total reflection," Journal of Applied Physics, vol. 115, no. 1, Article ID 013104, 2014.

[4] E. A. Marcatili, "Dielectric rectangular waveguide and directional coupler for integrated optics," Bell System Technical Journal, vol. 48, no. 7, pp. 2071-2102, 1969.

[5] J. E. Heebner, R. W. Boyd, and Q. H. Park, "SCISSOR solitons and other novel propagation effects in microresonator-modified waveguides," Journal of the Optical Society of America B: Optical Physics, vol. 19, no. 4, pp. 722-731, 2002.

[6] M. K. Park, J. Q. Yiying, J. S. Kee et al., "Silicon microring resonators for label-free aptamer-based biosensing," in Proceedings of the 7th IEEE Conference on Industrial Electronics and Applications (ICIEA '12), pp. 1599-1602, July 2012.

[7] X.-M. Lv, Y.-Z. Huang, Y.-D. Yang et al., "Influences of carrier diffusion and radial mode field pattern on high speed characteristics for microring lasers," Applied Physics Letters, vol. 104, no. 16, Article ID 161101, 2014.

[8] M. Rudé, J. Pello, R. E. Simpson et al., "Optical switching at $1.55 \mu \mathrm{m}$ in silicon racetrack resonators using phase change materials," Applied Physics Letters, vol. 103, no. 14, pp. 25402545, 2013.

[9] X. Zhao, J. M. Tsai, H. Cai et al., "A nano-opto-mechanical pressure sensor via ring resonator," Optics Express, vol. 20, no. 8, pp. 8535-8542, 2012.

[10] B. Bhola and H. W. Steier, "A novel optical microring resonator accelerometer," IEEE Sensors Journal, vol. 7, no. 12, pp. 17591766, 2007.

[11] F. Qiu, A. M. Spring, F. Yu, and S. Yokoyama, "Complementary metal-oxide-semiconductor compatible athermal silicon nitride/titanium dioxide hybrid micro-ring resonators," Applied Physics Letters, vol. 102, no. 5, Article ID 051106, 2013.

[12] J. Lu, J. Pan, S. S. Yeh et al., "Negative correlation between charge carrier density and mobility fluctuations in graphene," Physical Review B, vol. 90, no. 8, Article ID 085434, 2014.

[13] L. Sapienza, H. Thyrrestrup, S. Stobbe, P. D. Garcia, S. Smolka, and P. Lodahl, "Cavity quantum electrodynamics with anderson-localized modes," Science, vol. 327, no. 5971, pp. 1352 $1355,2010$.

[14] W. Hwang, H. Tao, and D. H. Kim, "A physically transient form of silicon electronics," Science, vol. 337, no. 16, p. 1640, 2012.

[15] H. F. Zhang, Y. J. Zhao, and F. Tao, "Five layer symmetrical man-made metamaterials structure of surface plasmons," Acta Photonica Sinica, vol. 39, no. 12, pp. 2234-2240, 2010.

[16] X.-T. Kong, W.-G. Yan, Z.-B. Li, and J.-G. Tian, "Optical properties of metal-multi-insulator-metal plasmonic waveguides," Optics Express, vol. 20, no. 11, pp. 12133-12146, 2012.

[17] Q. Z. Li, X. Y. Li, Y. C. Sun et al., "Free spectral range of double single micro-ring resonant filter," Acta Optica Sinica, vol. 32, no. 7, pp. 224-229, 2012. 

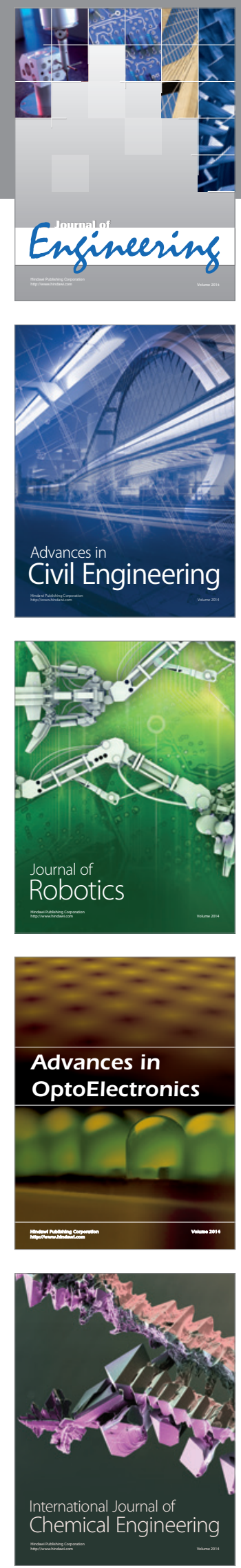

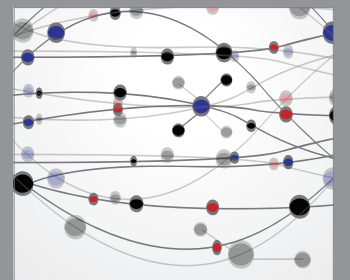

The Scientific World Journal
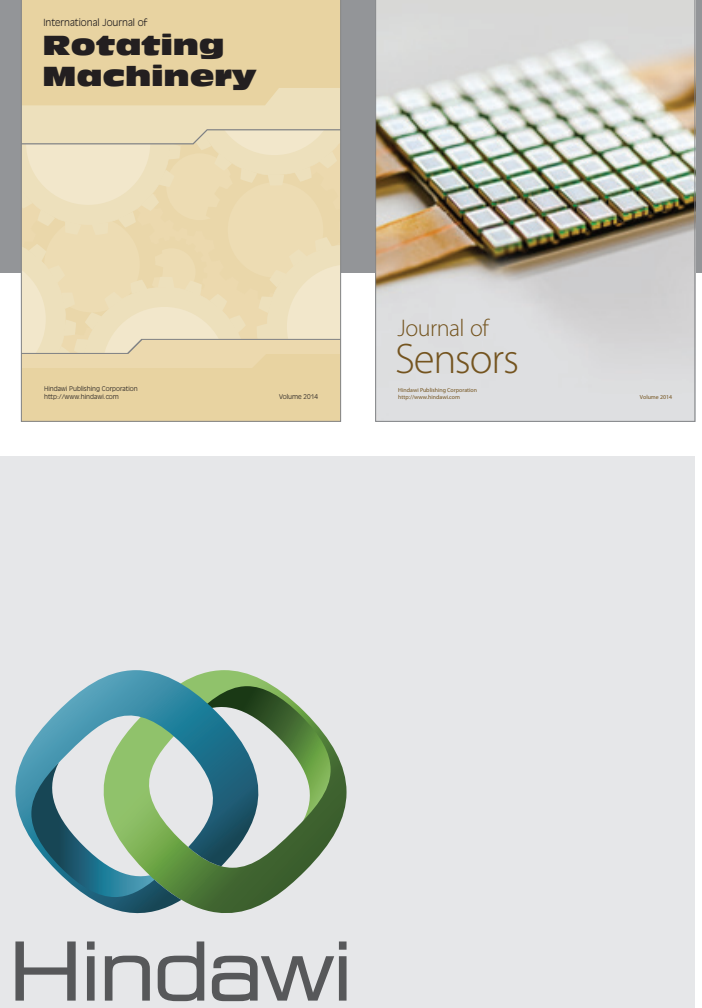

Submit your manuscripts at http://www.hindawi.com
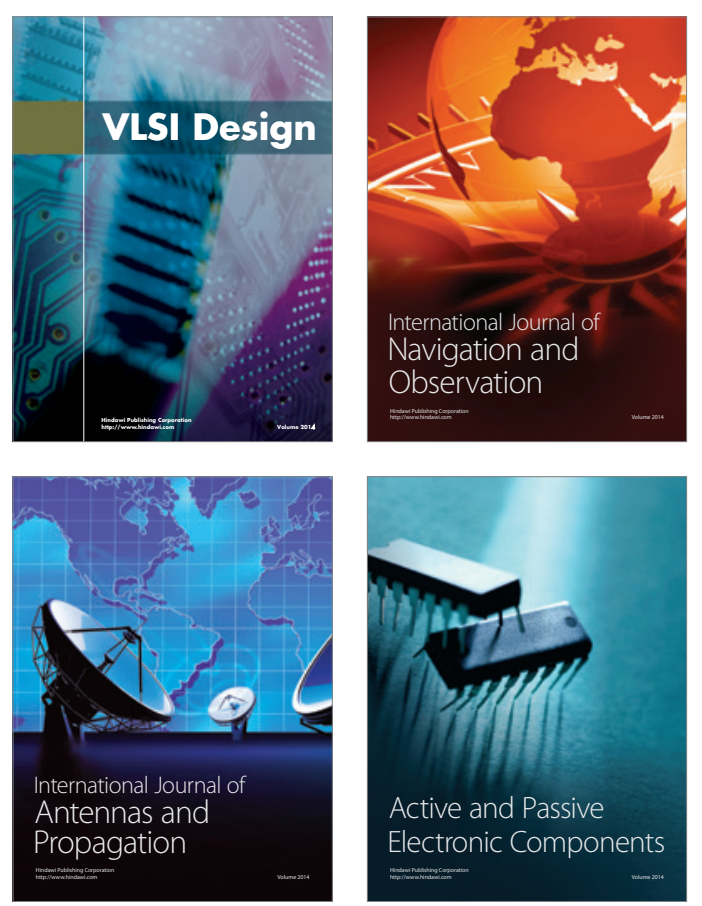
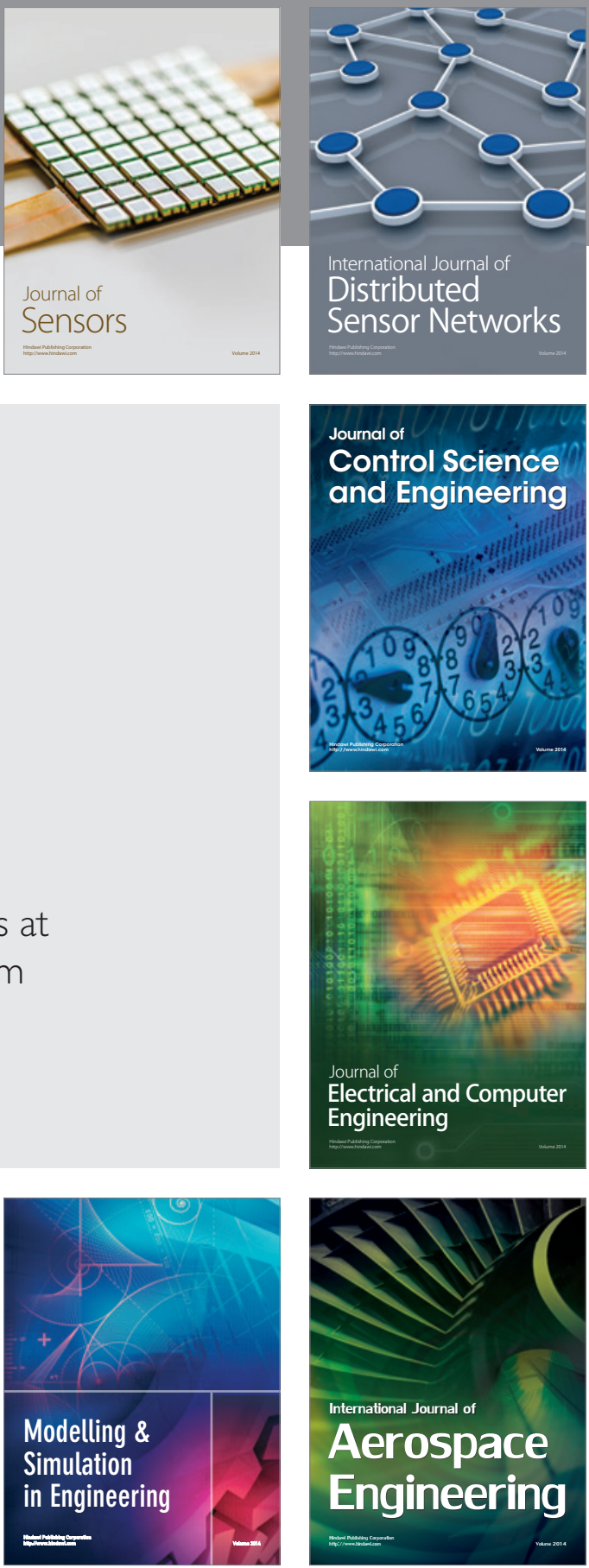

Journal of

Control Science

and Engineering
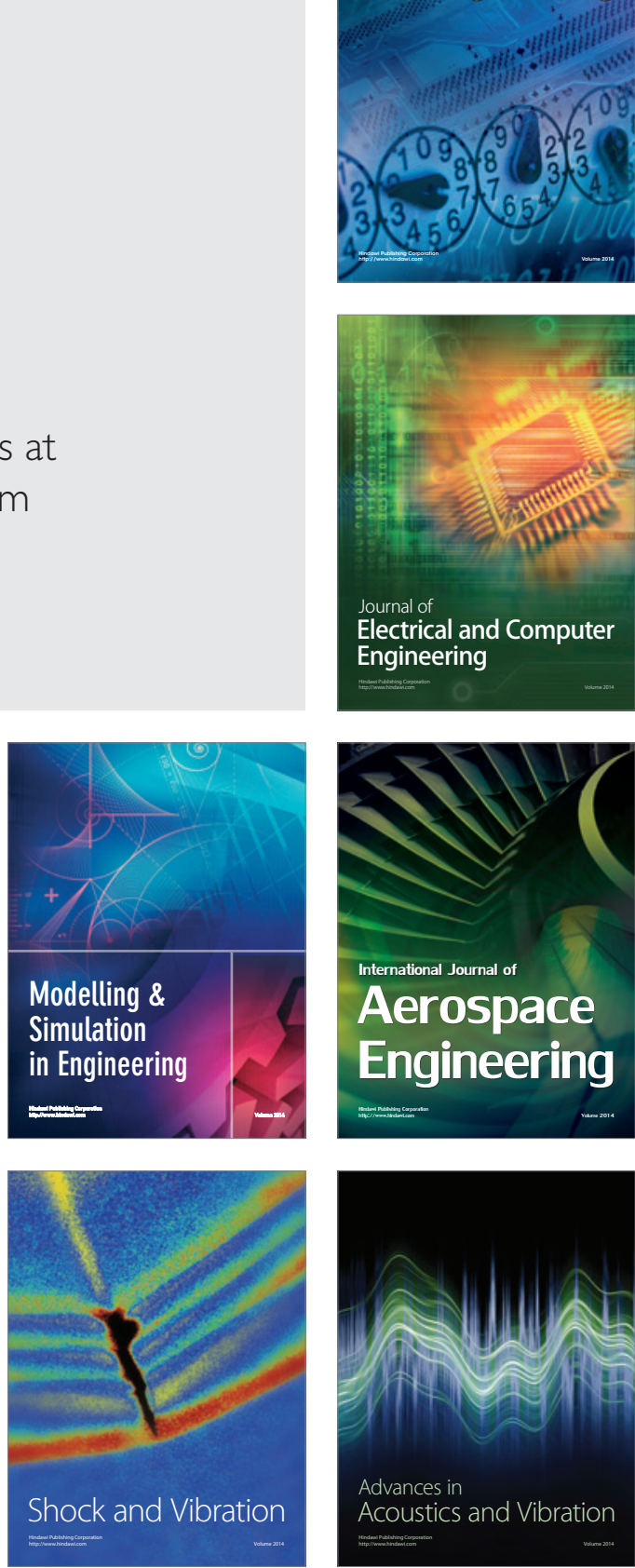